\title{
HexA is required for growth, aflatoxin biosynthesis and virulence in Aspergillus flavus
}

\author{
Jun Yuan ${ }^{\dagger}$, Ding Li ${ }^{\dagger}$, Ling Qin, Jiaojiao Shen, Xiaodong Guo, Elisabeth Tumukunde, Mingzhu Li \\ and Shihua Wang*
}

\begin{abstract}
Background: Woronin bodies are fungal-specific organelles whose formation is derived from peroxisomes. The former are believed to be involved in the regulation of mycotoxins biosynthesis, but not in their damage repair function. The hexagonal peroxisome protein (HexA or Hex1) encoded by hexA gene in Aspergillus is the main and the essential component of the Woronin body. However, little is known about HexA in Aspergillus flavus.

Results: In this study, hexA knock-out mutant ( $\triangle$ hexA) and complementation strain ( $\triangle$ hex $A^{C}$ ) were produced using homologous recombination. The results showed that, $\triangle$ hexA and $\triangle$ hexA $A^{C}$ were successfully constructed. And the data analysis indicated that the colony diameter, stress sensitivity and the sclerotia formation of $A$. flavus were nearly not affected by the absence of HexA. Yet, the deletion of hexA gene reduced the production of asexual spores and lessened virulence on peanuts and maize seeds markedly. In addition, it was also found that there was a significant decrease of Aflatoxin B1 production in deletion mutant, when compared to wild type.
\end{abstract}

Conclusions: Therefore, it suggested that the hexA gene has an essential function in conidia production and secondary metabolism in A. flavus. The gene is also believed to be playing an important role in the invasion of $A$. flavus to the host.

Keywords: hexA, Aspergillus flavus, Aflatoxins, Virulence

\section{Background}

Over the past few years, the food security crisis has been a burning subject all over the world. This problem was not only affecting the human beings' health, but also closely affected the development of the human society at large. Therefore, a saprophyte fungus Aspergillus flavus, was known as a main contaminant of crops. A. flavus can infect a variety of major economic crops, such as peanuts, corns, cottonseed and nuts, resulting in extremely serious food safety problems [1-3]. According to a report by the Food and Agriculture Organization of the United Nations, $25 \%$ of grain supplies in the world should not be consumed due to mycotoxin contamination which is

\footnotetext{
*Correspondence: wshyyl@sina.com

† Jun Yuan and Ding Li contributed equally to this work

Key Laboratory of Pathogenic Fungi and Mycotoxins of Fujian Province,

Key Laboratory of Biopesticide and Chemical Biology of Education

Ministry, and School of Life Sciences, Fujian Agriculture and Forestry

University, Fuzhou 350002, China
}

mainly caused by $A$. flavus $[4,5]$. On the other hand, $A$. flavus is the second major cause of invasive aspergillosis in humans [6]. The excess of A. flavus spores inhalation or contact with contaminated crops may cause a plurality of parts of the human lung aspergillosis and skin, nails and other infection [7]. These infections are mainly prevalent in areas with high temperatures, such as the Middle East, where the fungus is the most common cause of sinusitis and keratitis [8]. Under appropriate conditions, A. flavus will produce a variety of highly toxic and carcinogenic secondary metabolites, such as aflatoxin (AF) and cyclopiazonic acid. The World Health Organization (WHO) has designated aflatoxins as a natural existence of class I carcinogen. Due to stable physical and chemical properties of aflatoxin, simple processing can not get rid of the yellow aspergillus toxin. Therefore, it is very easy to take aflatoxins from contaminated crops into the food chain, resulting in more wide range of contamination and unavoidable economic losses [9]. 
In order to resist various environmental stresses and injuries from the outside, filamentous fungi developed a set of damage repair mechanisms in the evolution. In 1864, Woronin body in Ascobolus pulcherrimus was first described by a Russian scientist Michail Stepanowitsch Woronin [10]. It was then, named by Buller few years later [11]. After that, this organelle was found to be blocking the septal pores in case of damage in a variety of ascomycetes [12-15].

Woronin body has a dense-core consisting of HexA and WSC (woronin sorting complex) [16]. Hence, Hex orthologs in the Woronin body of filamentous fungi played essential roles in the conidial synthesis, fungal resistance $[14,17,18]$ and even the fungal virulence [19]. In this study, the homolog of Woronin body core protein found in A. flavus, HexA was identified and characterized in order to explore the function of HexA in aflatoxin biosynthesis and development in A. flavus.

\section{Results}

\section{Sequence analysis of HexA in A. flavus}

HexA protein sequences from different fungi species were downloaded from National Center for Biotechnology Information (NCBI) using "HexA" as the key words. A phylogenetic tree was constructed using the neighborjoining method by Mega 6.0. It was found that HexA protein was highly conserved in Aspergillus. The comparison of amino acid sequences revealed that HexA in A. flavus shown the highest identity (83\%) to $A$. oryzae, and had the lowest identity (65\%) to $A$. kawachill and A. luchuensis (Fig. 1a). Protein sequence analysis demonstrates that HexA protein contains a conserved domain, named S1_ Hex1 from 419 to 493 (Fig. 1b). This domain often presented in the HexA protein of fungi suggests that HexA should be a component of Woronin body in A. flavus. Meanwhile, it was confirmed that there is only a single copy of the hexA gene in the genome by analyzing the published genome sequences of $A$. flavus NRRL3357.

\section{Generation of $A$. flavus hexA deletion mutants and complementation}

In order to identify the potential biological function of HexA in A. flavus, deletion mutants of the hexA gene were constructed using pyrG selection. The schematic diagram of homologous recombination strategy is shown in Fig. 2a. Knockout mutant $\Delta$ hexA was performed using uracil/uridine autotrophy transformants. Afterward, the gene deletion was verified by $\mathrm{qPCR}$ with $\mathrm{CDNA}$ as a template. The result showed that a 187 bp DNA fragment from the ORF of hexA was amplified from WT and $\Delta$ hex $A^{\mathrm{C}}$ by qPCR but not from $\Delta$ hexA mutant (Fig. 2b). Also, it indicated there was no other homologous gene in the genome. Then the mutant was selected to carry out further study. After inoculated on YES and PDA agar medium for 4 days, the colony phenotypes of the $\Delta$ hex $A$ mutant was observed. The results showed that there was little difference of colony morphology between the mutant, WT and complementation strains, when they grew on PDA and YES media (Fig. 2c).

\section{Effects of HexA on the conidia production of $A$. flavus}

After grew on YES and PDA agar medium for 5 days and transfer, the conidiophores of strains were produced and surveyed by electron microscope (Fig. 3a). It was found that in the mutant $\Delta h e x A$, conidiophores developed imperfectly. The length became much shorter and only a small part of them developed completely. The density and the number of conidia at the top of the conidiophores were significantly decreased. While the conidiophores of $\mathrm{WT}$ and $\Delta h e x A^{\mathrm{C}}$ was very long, intensive and complete. Also, the data analysis indicated that there was a significant decrease in the conidia production of the knockout strains when compared to the WT or complementation in both the PDA medium and YES medium (Fig. 3b). All these results implied that hexA gene might regulate the conidiophore development and the loss of the gene would lead to the reduction of the conidia in A. flavus.

\section{Effects of hexA deletion on sclerotia formation}

In order to study whether hexA gene was related to the sclerotial production, WT, $\Delta$ hexA and $\Delta h e x A^{\mathrm{C}}$ were inoculated on the sclerotial inducing Wickerham's solid medium (WKM) at $37{ }^{\circ} \mathrm{C}$ for 7 days in dark. The quantity of sclerotium was calculated after washing the media with $75 \%$ alcohol. As shown in Fig. 4a, under a microscope, the colony center of the mutant became much thicker than that of WT and $\Delta h e x A^{\mathrm{C}}$. And the statistics data indicated there was no significant difference of the sclerotium number between the mutants and WT $(\mathrm{P}>0.05)$ (Fig. 4b).

\section{Effects of HexA on aflatoxin biosynthesis in A. flavus}

Aflatoxin is one kind of well-known secondary metabolite of saprophytic fungus $A$. flavus. The production of toxin was associated with the virulence of $A$. flavus to some extent. To determine the effect of hexA on AF biosynthesis, thin layer chromatography (TLC) was carried out to test AF production in the WT, $\Delta$ hexA and $\Delta h e x A^{\mathrm{C}}$ after cultivated in liquid YES for 6 days (Fig. 5a). The quantitative analysis results demonstrated that Aflatoxin $\mathrm{B}_{1}\left(\mathrm{AFB}_{1}\right)$ in the $\Delta$ hex $A$ was significantly decreased. The quantitative analysis manifested that amount of $\mathrm{AFB}_{1}$ produced by the mutant strains were less than $50 \%$ of the WT and $\Delta h e x A^{\mathrm{C}}$ (Fig. $5 \mathrm{~b}$ ). Therefore, it indicated that hexA gene played an important and positive role in regulating AF production in A. flavus. 


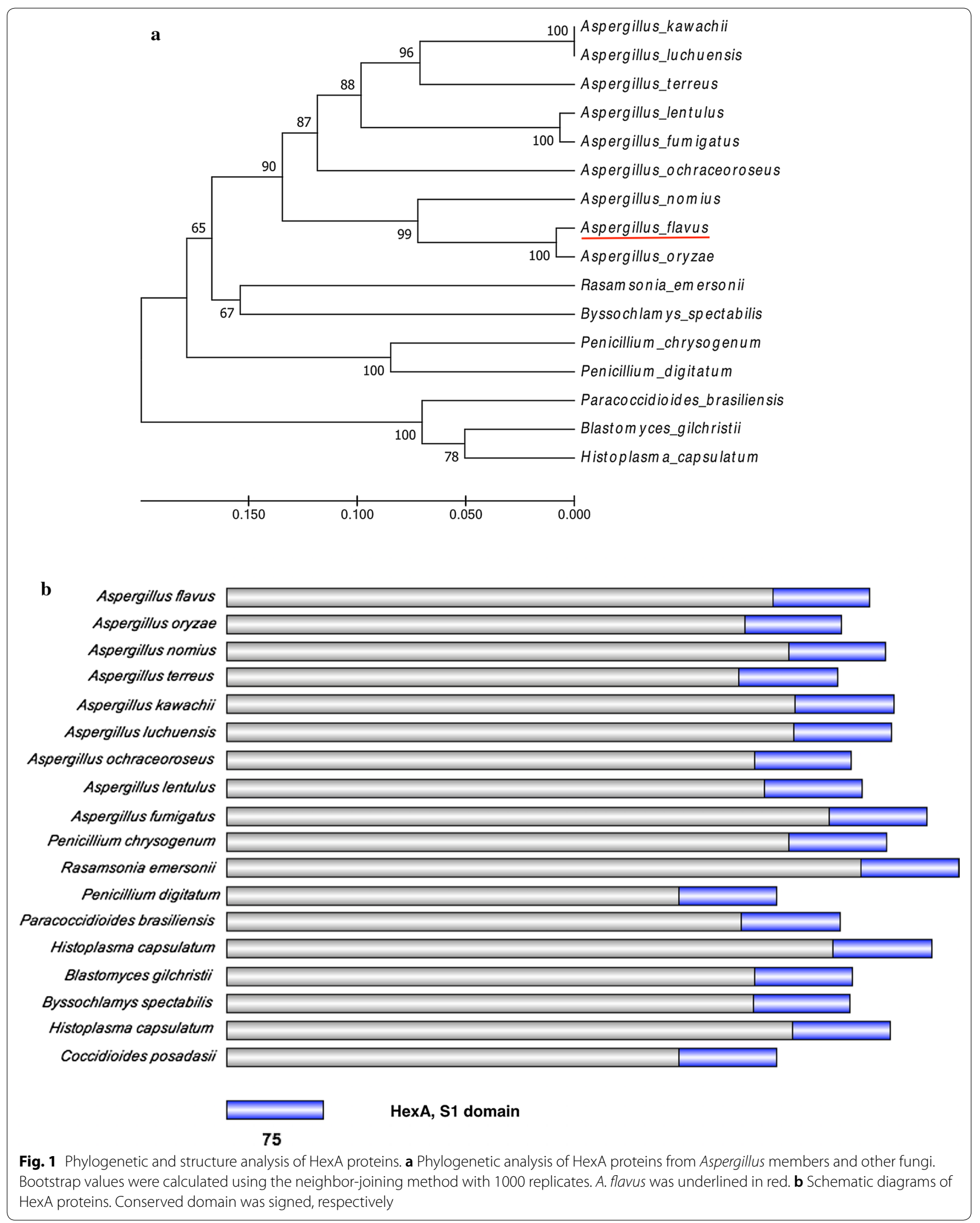




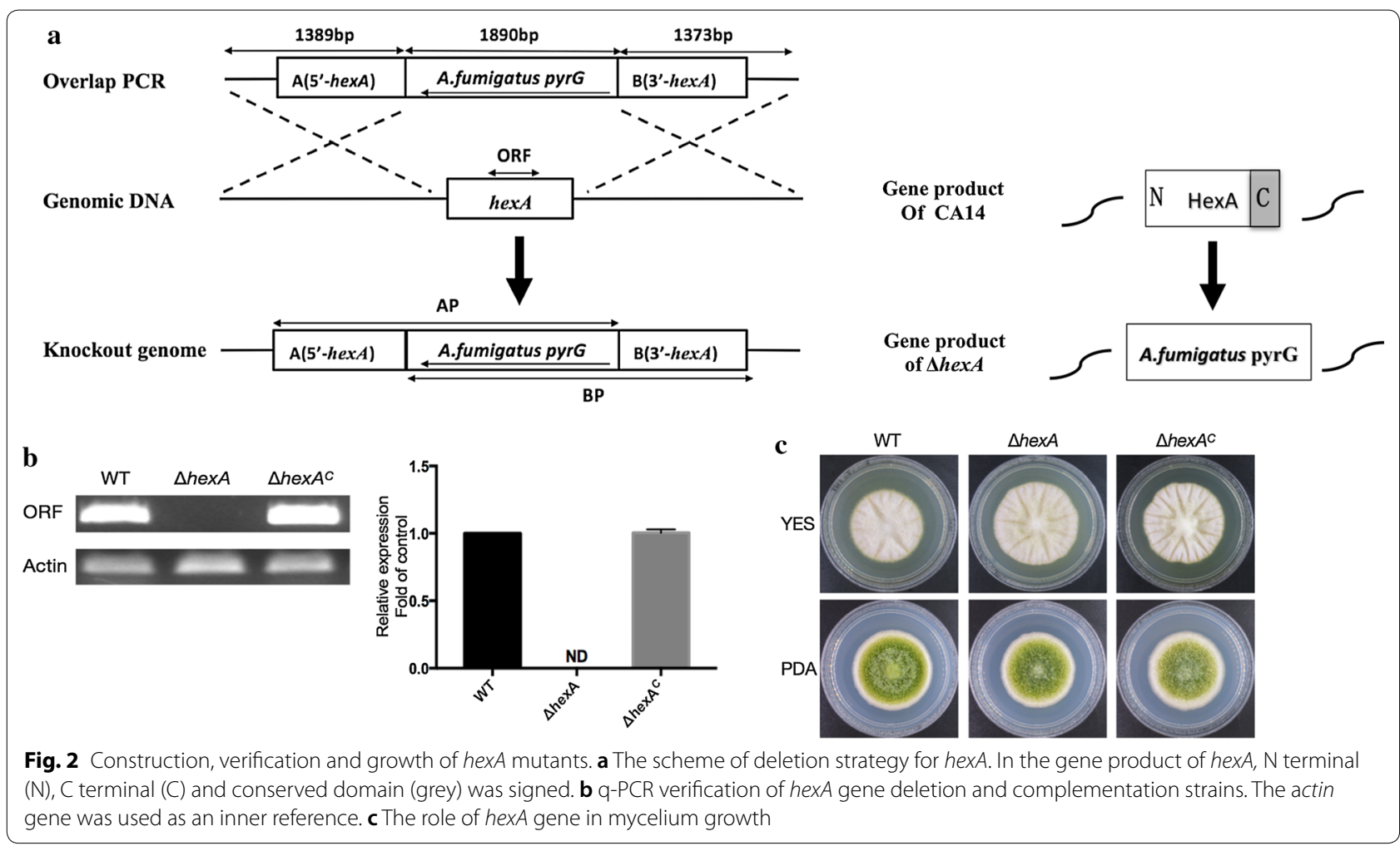

In order to further verify the function of HexA in modulating secondary metabolite production, and subsequently, the transcription levels of genes that are relevant to aflatoxin biosynthesis were measured by qPCR. In accordance with aflatoxin production, the $\mathrm{qPCR}$ results suggested that the loss of hexA gene attenuated almost all the transcripts of candidates (Fig. 5c). Among them, included a global transcription factor, aflR in the upstream of aflatoxin biosynthesis pathway and a collaborative regulatory gene, aflS. Meanwhile, the structure genes in toxin biosynthesis cluster which encoding the key enzymes in the downstream were also detected. By contrast, the expression of $a f l K$ and $a f l D$ were severely suppressed compared with the WT and $\Delta h e x A^{\mathrm{C}}$. These results showed that HexA might paly an important role in $\mathrm{AF}$ biosynthesis by regulating expression of some genes in AF cluster.

\section{Effects of HexA on stress response of $A$. flavus}

Woronin body of fungi might play a role in stress resistance of strain according to the previous publications. To this end, several environmental factors such as hyperosmotic stress and cell wall damaging reagent were tested in the study. The morphology of colony was observed after growing up for 5 days (Fig. 6a). Firstly, it could be observed that there was no significant difference in terms of growth between the $\Delta h e x A$ mutant, WT and $\Delta h e x A^{\mathrm{C}}$ in the presence of $\mathrm{NaCl}$. Results also suggest that $\Delta$ hex $A$ mutants were not sensitive to cell-wall damaging agents, Congo red, Calcofluor white and lauryl sodium sulfate (SDS) (Fig. 6a, b). Contrary to previous reports of other fungi [19], these results indicated that HexA was probably not involved in responses to specific stress conditions in A. flavus.

\section{Effects of HexA on pathogenicity to seeds}

Aspergillus flavus is considered to be one of the most common polluting fungi of the crops. To detect if the deletion of hexA might affect seed infections, in this study, the ability of $A$. flavus to infect peanut seeds and maize seeds was tested by colonizing them with $\Delta$ hexA mutants, WT and $\Delta h e x A^{\mathrm{C}}$, respectively. Results indicated that the Mock group had not been infected by A. flavus, which showed that the prophase treatment and negative control was successful. At the same time, it was clearly found that skins of peanut and maize seeds have been completely colonized with fluffy mycelium in the WT and $\Delta h e x A^{\mathrm{C}}$ Also a large number of deep green conidia were produced in these strains, compared to the $\Delta h e x A$ mutants (Fig. 7a, e). The number of the conidia significantly decreased on peanut and maize seeds inoculated with deletion mutants compared to WT and $\Delta h e x A^{\mathrm{C}}$ (Fig. 7b, f). The production of aflatoxin was equally altered in the mutants. Obtained data demonstrated that 

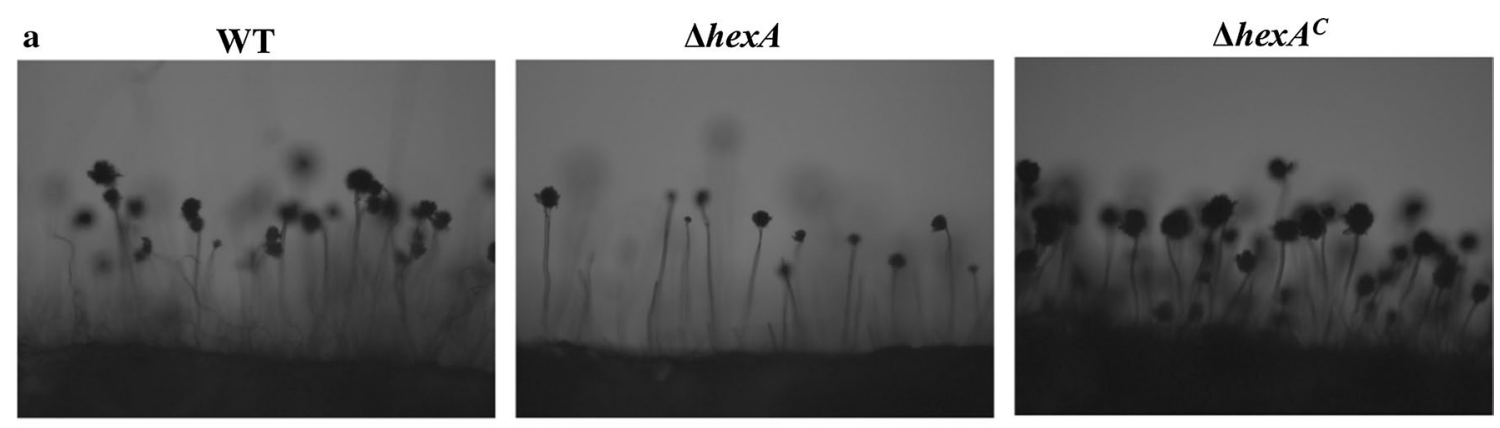

b

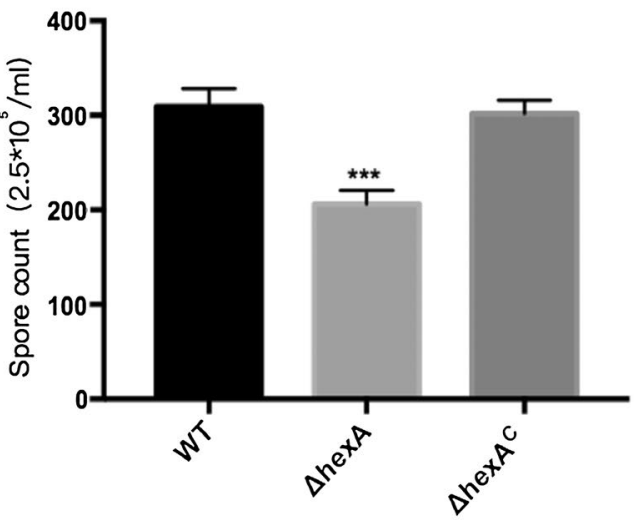

PDA

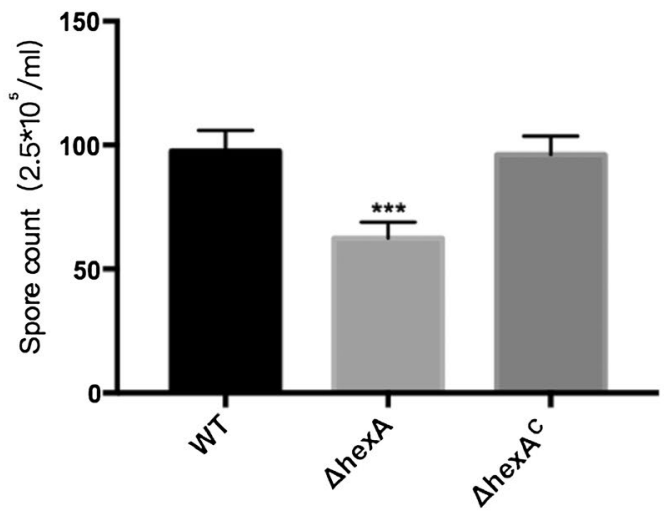

YES

Fig. 3 Conidia production is attenuated in null hexA mutants. a The electron micrograph of conidiophores of WT, $\triangle$ hexA and $\triangle$ hexA $A^{C}$. Strains were pre-cultured on PDA and Yes were transferred on sterile slides under $37^{\circ} \mathrm{C}$ overnight to induce conidiophores. (Magnification scale: $\times 200$ ) b The number of conidia in WT, $\triangle$ hexA and $\triangle$ hexA $A^{C}$ strains was measured after grown on YES and PDA agar for 5 days at $37^{\circ} \mathrm{C}$. Values are a mean of four replicates. ${ }^{* *}$ Significant difference between the WT and mutant strains at $\mathrm{P}<0.001$, as assessed by one-way ANOVA and Dunnett's multiple-comparisons test

toxin biosynthesis reduced distinctly in A. flavus mutants without hexA on peanuts (Fig. 7c, d) and maize seeds (Fig. 7g, h). All these results above suggested that HexA in A. flavus took part in the colonization and pathogenicity to the host.

\section{Discussion}

As we all know, a special and unique organelle named "Woronin body" existed in filamentous ascomycete and deuteromycete fungi. It could seal the septal pore when cells countered the cell wall damage factors. According to the researches reported previously, Hex protein was a key part of Woronin body. The hexA gene was first found to be responsible for encoding the main component of the Woronin body in Neurospora crassa by Jeddand Chua [14]. As a result, Hex protein was given much attention for its quickly repairing function in attenuating the loss of cytoplasm and maintaining cellular integrity. Afterwards, this protein was also identified playing important roles in the other fungi genera in succession, such as Aspergillus [20], Fusarium [21], Trichoderma [22] and Arthrobotrys [23]. In this study, we identified the hexA gene in A. flavus, and bioinformatics analysis indicated that HexA in A. flavus was ortholog of Woronin body major protein found and identified in other members of Aspergillus. Yuan et al. [15] confirmed that the structural organization of Hex1 in $N$. crassa was similar with eIF-5A in P. aerophilum and Methanococcus jannaschii. They suggested that hexA gene might have been evolved through duplication of an ancestral eIF-5A gene and gained additional features. In the present study, multiple amino acid alignment (data not shown) and conserved region analysis showed that the C-terminal of the HexA proteins in Aspergillus was relatively conserved. Similar with hex 1 existed in $N$. crassa, the sequence from 318 to 488 of HexA in A. flavus hitted a domain of eukaryotes translation initiation factor eIF-5A, which revealed that HexA was related with eIF-5A in the long-term evolution. But different from the eIF-5A, the sequence of HexA in A. flavus possessed a peroxisome target signal site in the $\mathrm{C}$-terminus and a very divergent $\mathrm{N}$-terminus, which suggested that HexA proteins were also related to peroxisomes and might have essential impact on the 
a
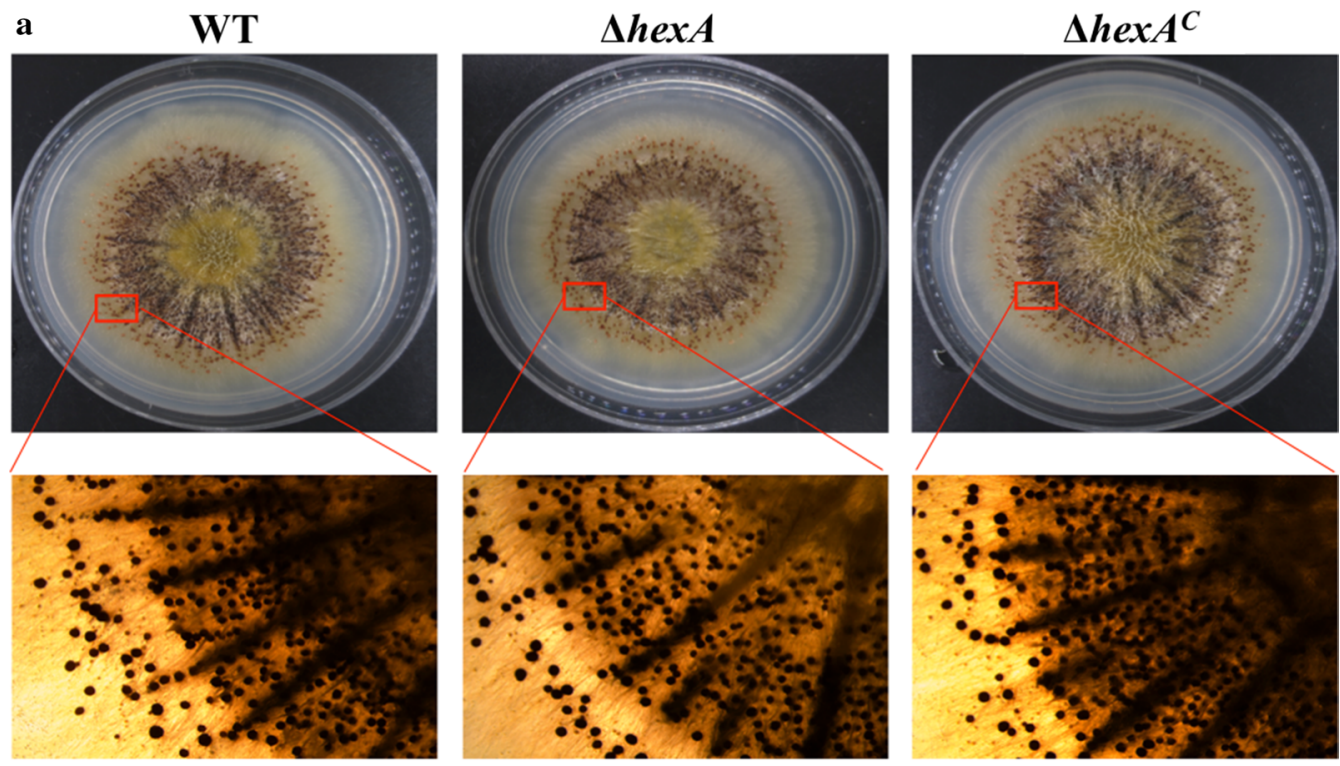

$\mathbf{b}$

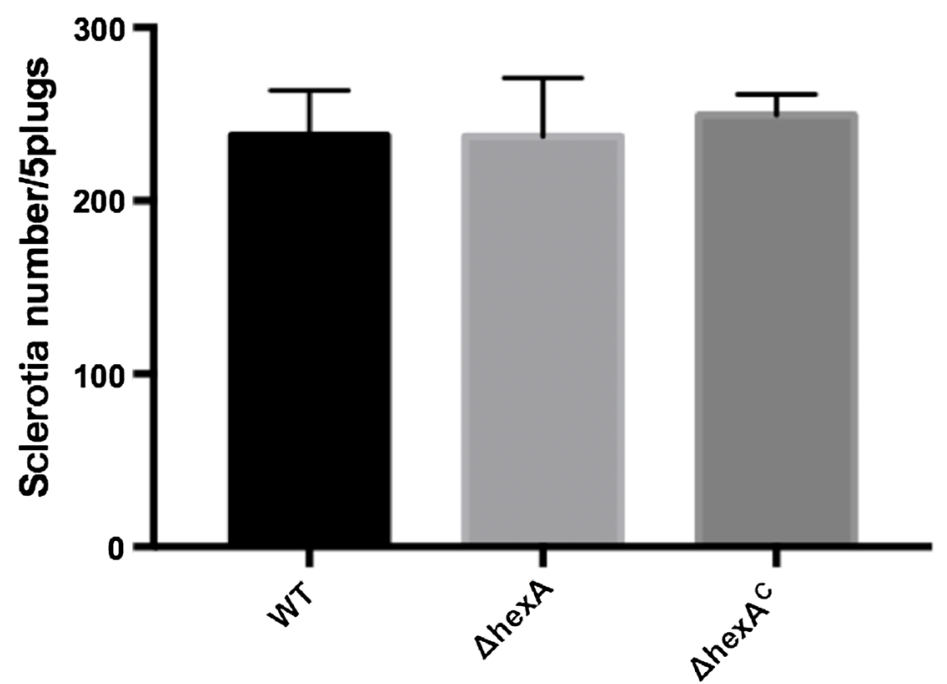

Fig. 4 Phenotype analysis of hexA deletion, WT and $\triangle$ hexA $A^{C}$ strains on sclerotia production. a Morphology of strains on sclerotia-inducing WKM medium after being washed by $75 \%$ ethanol and their detail views. $\mathbf{b}$ The number of sclerotia in WT, $\triangle$ hexA and $\triangle$ hex $A^{\mathrm{C}}$ strains was measured after grown on WKM medium for 7 days at $37^{\circ} \mathrm{C}$ in dark

other aspect of fungal development and physiological activities, except the partial function as eIF-5A.

Ever since the discovery of Hex1 in N. crassa [24], several studies have shown that Hex protein was not only an important part of Woronin body conserved in other filamentous fungi systems playing a similar role in controlling the cargo molecules transport from the septal pore. Studies also showed that Hex protein played an essential part in the development and virulence regulation of pathogenic fungi. However, the function performed by HexA in pathogen A. flavus is largely unknown. Hence, additional studies on the functions of Hex protein are needed to determine by series experiments. Hence, this study focuses determining the role of hexA gene in $A$. flavus in both development modulation and AFs biosynthesis. In $F$. graminearum, the research by Son et al. [21] showed that the lack of Hex1 impaired conidiation and conidial viability. In our study, in two different media (PDA and YES), although there was no clear growth inhibition detected between mutants, WT and $\Delta h e x A^{\mathrm{C}}$, it was, however, found that the loss of HexA resulted in the development of defect conidiophores and suppression of 


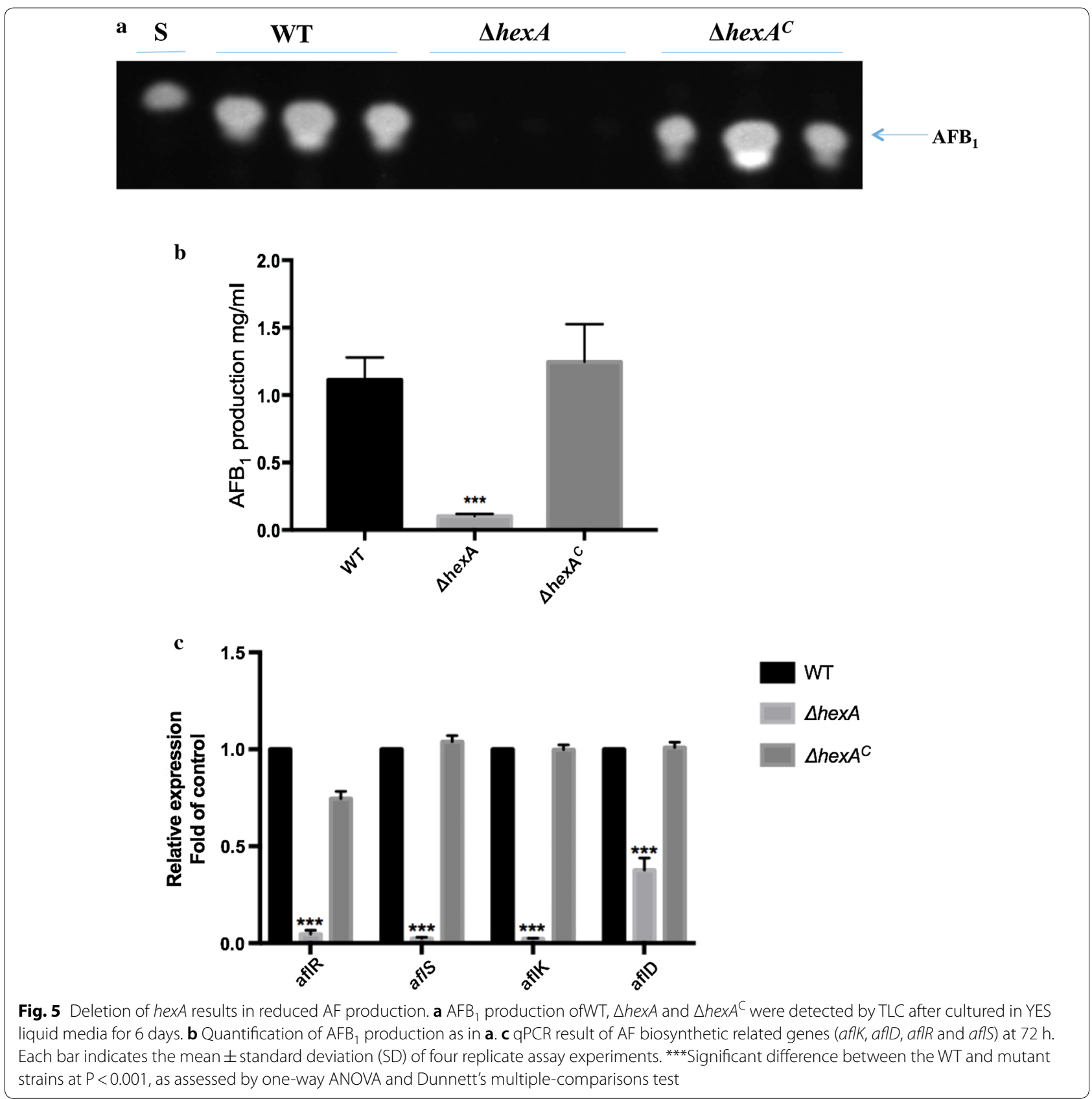

conidia production. The above implies that HexA may contribute to cell division and asexual development in A. flavus. The similar phenotypes assessed in A. oligospora, a famous nematode-trapping fungus. Its mutant strain without AoHex 1 also showed compromised conidiation [23]. The above-indicated studies suggested that HexA was one of the key members in regulating the asexual reproduction in fungi. As one of the most toxic and carcinogenic natural contaminants, AFs are natural polyketide-derived mycotoxins and biosynthesized by an extremely complicated mechanism. Surprisingly, to the best of our knowledge, we, for the first time, found that HexA was related to secondary metabolism in fungi. Our result showed that $A_{F B}$ biosynthesis was significantly blocked in $\Delta$ hexA mutants, suggesting that the deletion of hexA might result in additional defects and hence affects the aflatoxin production. The qPCR provided further evidence that the absence of HexA down-regulated the expression of genes in aflatoxin biosynthesis cluster indeed. As well as the regulator genes (aflR and aflS), the 


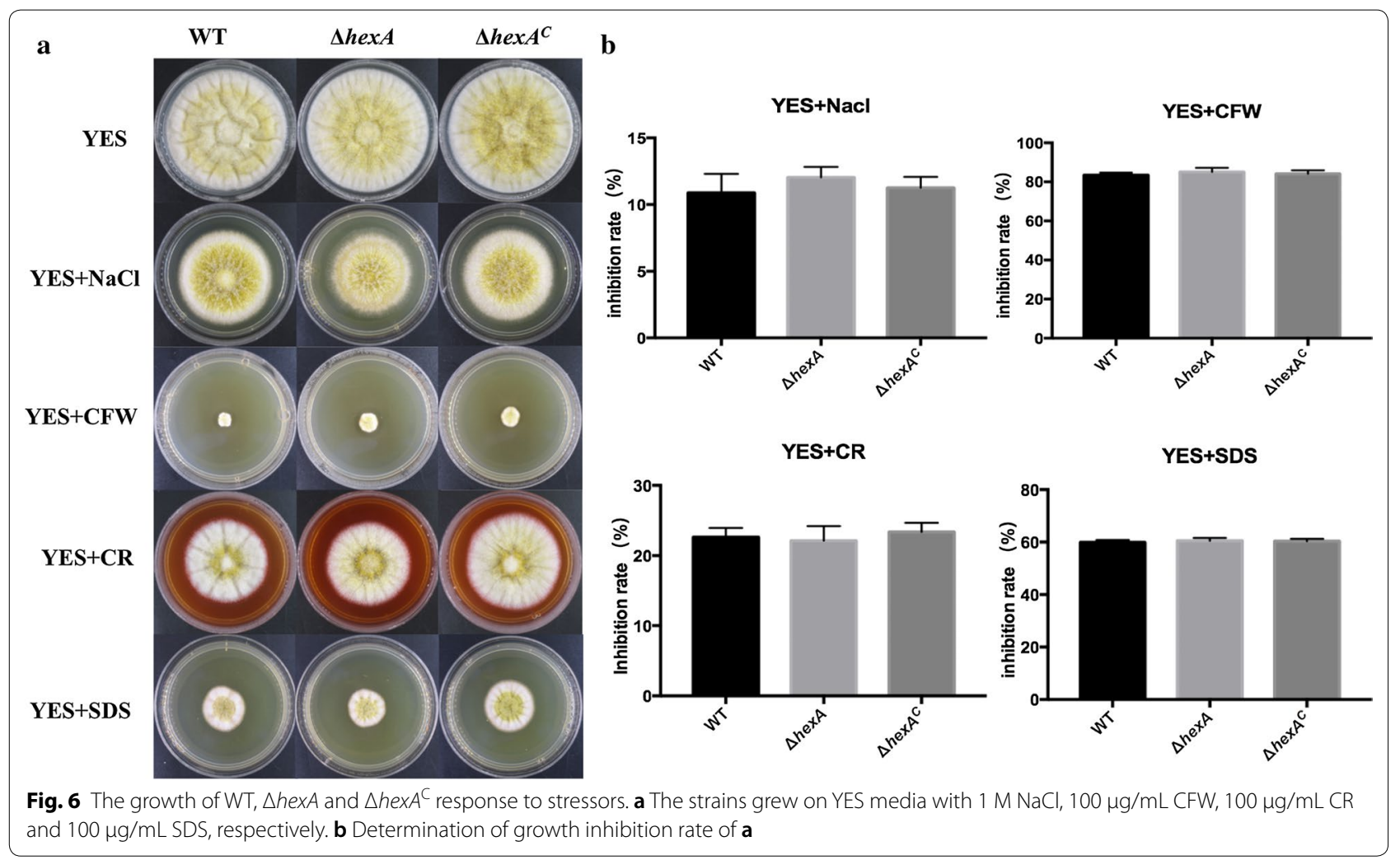

transcription levels of $a f l K$ and $a f l D$ genes were lowered to some extent. These genes encoded desaturase, and norsolorinic acid ketoreductase respectively, and responsible for the key conversion steps in aflatoxin biosynthesis pathway [25-27]. The deletion of hexA depressed the expression of regulatory gene and then modulated the genes in the aflatoxins producing cluster. All these studies indicated that HexA played important roles in regulating the asexual reproduction and exerted a sophisticated effect on the $\mathrm{AFB}_{1}$ biosynthesis in A. flavus.

Although as reported, there were no clear effects on growth in pathogenic mould $A$. fumigatus under oxidative and hyperosmotic stress in the absence of hexA, increased sensitivity were found in the presence of some regents related to integrity of the cell wall and membranes [19]. These results indicated that HexA in A. fumigatus participated in the maintenance of cellular integrity, which was always regarded as basic ability of Woronin body. Interesting, not consistent with the phenomenon above, in our study, no significant changes in sensitivity to stressors such as SDS, Calcofluor white and Congo red were observed between $\Delta$ hexA mutants and WT. Reports on Woronin body in other fungi showed that except close proximity to septal pores, this organelle sometimes present in nonseptal regions, such as the tips of the germlings and secondary infectious hyphae or at the cell periphery $[19,28-30]$. It suggested that the functions of Woronin body and HEX proteins should not be limited as a plug in emergency repair. The research in Trichoderma atroviride demonstrated that HEX1 participated in the protection of the cell membrane from dichlorvos stress by reducing excessive electrolyte leakage [31]. In addition, Son et al. reported that Hex proteins might be multifunctional gene. The FgHex1 in $F$. graminearum functioned in the synthesis of both strands of virus genomic RNA. Therefore, we speculated that hexA gene in A. flavus response to environment stress might function in a different pattern to other species [32].

(See figure on next page.)

Fig. 7 The effect of hexA gene on the pathogenicity of WT, $\triangle$ hexA and $\triangle$ hex $A^{C}$ of $A$. flavus. a The strains of the WT, $\triangle$ hexA and $\triangle$ hex $A^{C}$ were grown on peanut seeds at $28^{\circ} \mathrm{C}$ for 7 days. $\mathbf{b}$ Conidia production was assessed from the infected peanut seeds. c Quantification of AFB1 production as in d. $\mathbf{d}$ Aflatoxin was detected by TLC, which extracted from infected peanut seeds. e The strains of the WT, $\triangle$ hexA and $\triangle$ hexA $A^{C}$ were grown on maize seeds at $28^{\circ} \mathrm{C}$ for 7 days. $\mathbf{f}$ Conidia production was assessed from the infected maize seeds. $\mathbf{g}$ Quantification of AFB1 production as in $\mathbf{h}$. $\mathbf{h}$ Aflatoxin was detected by TLC, which extracted from infected maize seeds. ***The bars represent significantly different $(P<0.001)$ 

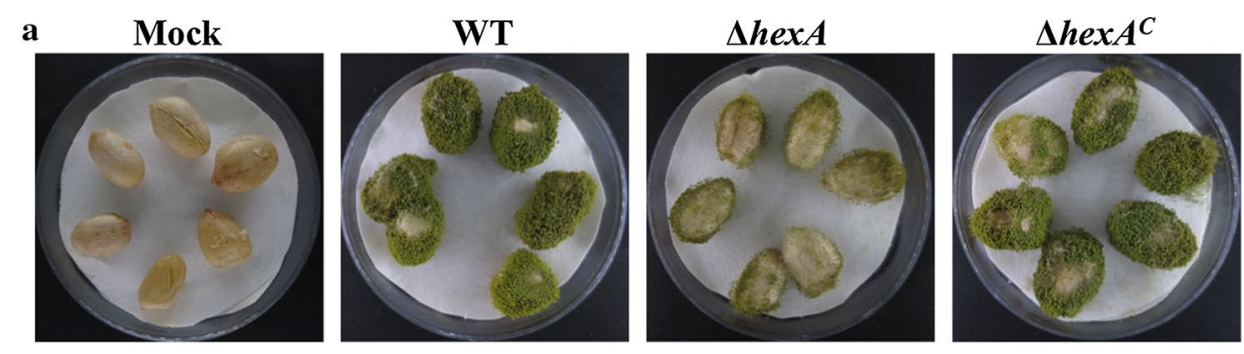

b
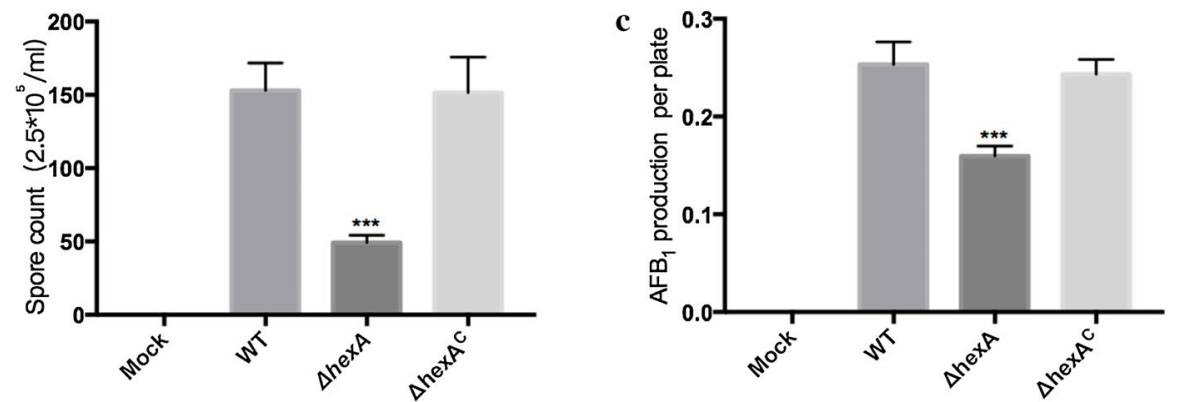

d
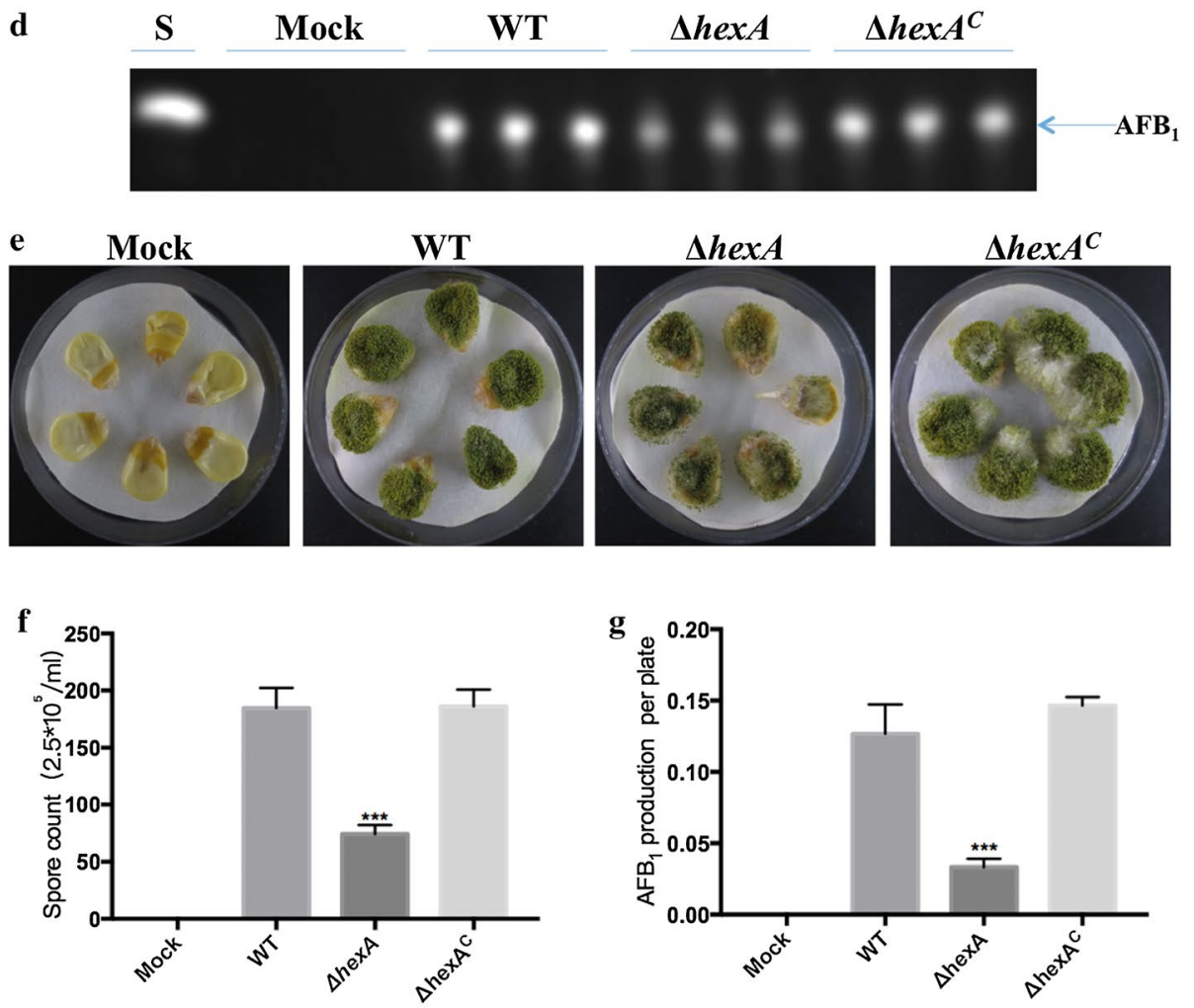

h

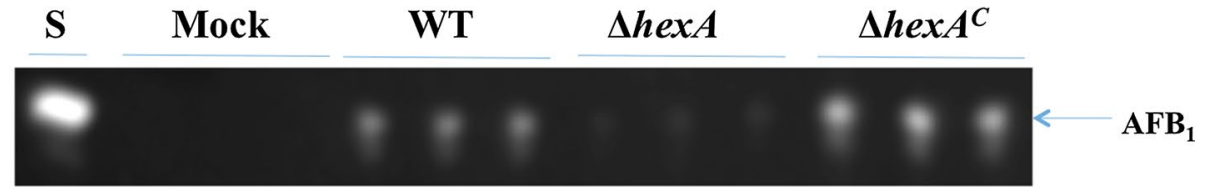


As we know, several recent studies revealed that Woronin body or Hex protein was also essential in efficient pathogenesis of fungal pathogens [32]. Soundararajan et al. [17] studied the function of Woronin body and Hex1, and found that in the graminaceous-parasitic fungus Magnaporthe grisea, no significant difference in morphology or growth rate was determined when Hex1 was absent, compared with the WT and $\Delta h e x A^{\mathrm{C}}$. While the infection ability of the strain reduced to about onefifth for absence of the Woronin body. To verify the pleiotropic effects of $\Delta$ hexA mutations that appeared on synthetic media, the virulence and toxin contribution of the HexA on peanuts were determined in our research. As a result, the deletion of hexA gene significantly decreased the pathogenicity on peanuts, which is in agreement with previous studies in A. fumigatus [19] and $M$. grisea [17]. Obtained data indicated that the hex $A$ gene played a direct role in the virulence of $A$. flavus.

\section{Conclusion}

In this study, we explored novel function of hexA gene in fungal pathogen A. flavus. We suggest that HexA can control the asexual processes and contribute to virulence on plant host. What is more, our results diversify the role of HexA, mostly known as a material sealing the septal pore of damaged cell. HexA is required for the production of $\mathrm{AFB}_{1}$ by regulating expression of some genes in AF biosynthesis cluster.

\section{Materials and methods}

\section{Strain and culture conditions}

The strain used in the study is listed in Table 1 [33]. A. flavus was cultured on YES, WKM or PDA media at $37^{\circ} \mathrm{C}$ or $28^{\circ} \mathrm{C}$.

\section{Phylogenetic tree generation}

HexA, a protein as a part of Woronin body of A. flavus was identified in the NCBI using the blast tool (http:// blast.ncbi.nlm.nih.gov/Blast.cgi). The phylogenetic tree was constructed with distinct HexA sequences from different species in Aspergillus genus and some other fungi using the Neighbour-joining method by MEGA6.0. Bootstrap analysis was performed with 1000 replicates.

Table 1 Wild-type and mutant strains used in this study

\begin{tabular}{lll}
\hline Strain & Genotype description & Reference \\
\hline A. flavus CA14PTs & $\triangle k u 70, \triangle p y r G$ & Nie et al. [33] \\
Wild-type (WT) & $\triangle k u 70$ & This study \\
$\triangle$ hexA & $\triangle k u 70, \triangle p y r G, \triangle$ hexA::pyrG & This study \\
\hline
\end{tabular}

\section{Construction of $\Delta$ hexA mutants and complementation}

Homologous recombination was used to generate the HexA mutant. All the primers used in this study were described in Table 2. To construct hexA mutants, a 1389 bp DNA fragment upstream from HexA was amplified with hexA-A-F and hexA-A-R primers. Then, a 1373 bp fragment downstream from HexA was amplified with hexA-B-F and hexA-B-R primers. P801-R and P1020-F were used to amplify the pyrG gene examining for the occurrence of homologous integration in strains. The upstream and downstream fragments with A. fumigatus pyrG marker were fused by overlap PCR previously described by Zhuang et al. [34]. To generate the hexA mutants, the fusion PCR product was transformed into protoplasts of the CA14 PTS $\Delta k u 70 \Delta p y r G$. The preparation of protoplasts and transformation was followed the established procedures described by Szewczyk et al. [35] and Yang et al. [36]. For gene complementation, the hexA ORF fragment was amplified from A. flavus and then transformed into the protoplasts of $\Delta$ hexA with $2 \mathrm{mg} / \mathrm{mL} 5$-FOA (5-fluoroorotic acid) to replace pyrG. After confirmed the removement of pyrG geneby PCR analysis was performed to that the pyrg gene was removed from the first step of complemented strains. Lastly, the pyrg gene was inserted behind the hexA to produce the pyrG prototroph complementation strains $\left(\Delta h e x A^{\mathrm{C}}\right)$ by using a homologous recombination. The complementation strain was tested using PCR analysis.

\section{Physiology experiments}

Conidial suspension ( $5 \mathrm{~mL}$ of a $10^{6}$ spore $/ \mathrm{mL}$ ) of mutant, WT and complementation strains was inoculated into YES and PDA media, respectively. The colony morphology was observed and the diameter of each colony was recorded after incubation for 4 days at $37{ }^{\circ} \mathrm{C}$. The conidial number was accounted by the haemocytometer after incubation for 5 days at the same temperature in YES and PDA media. At the same time, the strains were transferred on sterile slides under $37^{\circ} \mathrm{C}$ overnight to induce conidiophores. Then the conidiophores of strains were produced and surveyed by electron microscope (Magnification scale, $200 \times$ ). Sclerotium were induced in WKM medium and counted by method described in detail by Yang et al. [36]. For stress resistant analysis, conidia of $\Delta h e x A, \mathrm{WT}$ and $\Delta h e x A^{\mathrm{C}}$ strains $\left(10^{6}\right.$ spore $\left./ \mathrm{mL}\right)$ were spotted on YES media added with cell-wall perturbing agent $(100 \mu \mathrm{g} / \mathrm{mL}$ Calcofluor white, $100 \mu \mathrm{g} / \mathrm{mL}$ Congo red, $100 \mu \mathrm{g} / \mathrm{mL}$ SDS) or osmotic stress agent $(1 \mathrm{M} \mathrm{NaCl})$. All plates were incubated in dark at $37^{\circ} \mathrm{C}$ for 5 days, and growth status of colony was observed. 
Table 2 Primers used for the strain construction in this study

\begin{tabular}{|c|c|c|}
\hline Primers & Sequence $\left(5^{\prime}-3^{\prime}\right)$ & Description \\
\hline hexA-A-F & TCCTCGTAACTTTATCACCG & hexA deletion \\
\hline hexA-A-R & GGGTGAAGAGCATTGTTTGAGGCTTCGTTCTTCACGGGTTG & \\
\hline hexA-B-F & GCATCAGTGCCTCCTCTCAGACCTCAACGCCTCGTAAACA & \\
\hline hexA-B-R & GTGGCAGCAAGAGTATGG & \\
\hline hexA-ORF-F & TCCATTCAGCCAGCAACA & \\
\hline hexA-ORF-R & ACACGGGCGTCAAAGTCC & \\
\hline $\mathrm{PyrG} / \mathrm{F}$ & GCCTCAAACAATGCTCTTCACCC & HexA deletion (A. fumigatus pyrG) \\
\hline PyrG/R & GTCTGAGAGGAGGCACTGATGC & \\
\hline P801-R & CAGGAGTTCTCGGGTTGTCG & hexA mutant screen \\
\hline P1020-F & ATCGGCAATACCGTCCAGAAGC & \\
\hline hexA-O-F & TGGGTTATGCCTGTTCTGG & hexA mutant screen \\
\hline hexA-O-R & GGTTTCTTTCTCGGGTGC & \\
\hline Step1-F & GGGCAGTAGGTATTGTAGGT & hexA complementation \\
\hline Step1-R & CCTCGGTTCACTACAGCAC & \\
\hline ORF-UTR-F & TCAAGAGCCTACCTCATACC & \\
\hline ORF-UTR-R & GGGTGAAGAGCATTGTTTGAGGCACAAGCCACTTCCACCC & \\
\hline UTR-F & GCATCAGTGCCTCCTCTCAGACTCAACGCCTCGTAAACA & \\
\hline UTR-R & TGGCAGCAAGAGTATGG & \\
\hline Overlap-F & AGCCAGTCAACCCACCT & \\
\hline Overlap-R & CTTACGGCATTAGTACATTTCT & \\
\hline
\end{tabular}

\section{Pathogenicity tests}

To test the infection ability of the strains, pathogenicity tests were carried out by inoculating the kernels of peanuts and maize with WT, $\Delta h e x A^{\mathrm{C}}$ and $\Delta$ hexA. Peanuts and maize were pretreated according to the methods used by Zhang et al. [37]. Then the inoculated kernels were incubated in a plate with 3 layers of wet filter paper at $28{ }^{\circ} \mathrm{C}$ for 5 days.

\section{AF analysis}

In order to determinate whether HexA affect the production of AF in A. flavus, $10^{6}$ conidia of each strain were inoculated into $25 \mathrm{~mL}$ of liquid YES media in a $100 \mathrm{~mL}$ flask shaking at $180 \mathrm{r} / \mathrm{m}$ at $28{ }^{\circ} \mathrm{C}$ for 6 days. After that, AF was abstracted by chloroform and detected by thin layer chromatography (TLC) and UV light. $\mathrm{AFB}_{1}$ standard was purchased from Sigma (Sigma, Germany). The procedures of aflatoxin extraction and detection followed previously described methods [37].

\section{Real-time fluorescence quantitative reverse transcription PCR (qPCR)}

Mycelium of $\Delta$ hexA mutants, wild-type, and $\Delta h e x A^{\mathrm{C}}$ were harvested after incubated for $72 \mathrm{~h}$ in YES medium at $28{ }^{\circ} \mathrm{C}$ in the dark. RNA was extracted immediately by fully grinding in liquid nitrogen and using the Eastep
Total RNA Extraction Kit (Promega, USA). cDNA Synthesis and qPCR was performed using the methods described by Nie et al. [33]. Regulatory genes (aflR and aflS) and structural genes ( $a f K$ and $a f l D)$ were amplified by the primers list in Table 3. A. flavus actin gene was used as an endogenous control. The relative quantification of transcripts was calculated by the $2^{-\Delta \Delta \mathrm{Ct}}$ method [38]. qPCR assays were conducted with technical triplicates for each sample. And all experiments were repeated twice.

Table 3 Primers used for qPCR

\begin{tabular}{lll}
\hline Primers & Sequence $\left(\mathbf{5}^{\prime} \mathbf{- 3}^{\prime} \mathbf{)}\right.$ & Description \\
\hline hexA/QF & CTCTTCACCCGCCAACT & hexA qPCR \\
hexA/QR & CGCCCTGAGGAACAACT & \\
aflK/QF & GAGCGACAGGAGTACCGTAAG & aflK qPCR \\
aflK/QR & CCGATTCCAGACACCATTAGCA & \\
aflD/QF & TGTATGCTCCCGTCCTACTGTTC & aflD qPCR \\
aflD/QR & TGTAGTCTCCTTAGTCGCTTCATC & \\
aflS/QF & CGAGTCGCTCAGGCGCTCAA & aflS qPCR \\
aflS/QR & GCTCAGACTGACCGCCGCTC & \\
aflR/QF & AAAGCACCCTGTCTTCCCTAAC & aflR qPCR \\
aflR/QR & GAAGAGGTGGGTAGTGTTGTAG & \\
actin/QF & ACGGTGTCGTCACAAACTG & actin qPCR \\
actin/QR & CGGTTGGACTTAGGGTTGATAG & \\
\hline
\end{tabular}




\section{Abbreviations}

HexA: hexagonal peroxisome protein; $A F B_{1}$ : aflatoxin B1; AF: aflatoxin; WT: wild type; WKM: Wickerham's solid medium; TLC: thin layer chromatography; SDS: lauryl sodium sulfate.

\section{Authors' contributions}

SHW, JY and DL, designed the approach, collected data and interpreted the research results. SHW supervised DL and JY, prepared and submitted the manuscript. DL and LQ carried out phenotype detections. JJS, XDG and MZL provided technical assistance with $\mathrm{QPCR}$ and data analysis. ET participated in manuscript preparation and edition. All authors read and approved the final manuscript.

\section{Acknowledgements}

We thank Yuewei Yue for his effort in preparing the experimental materials. We also thank professor Feng Zhang and Dr. Kunlong Yang for their advice for this study.

\section{Competing interests}

The authors declare that they have no competing interests.

\section{Availability of data and materials}

The datasets used and/or analyzed during the current study available from the corresponding author on reasonable request.

\section{Consent for publication}

Not applicable.

\section{Ethics approval and consent to participate}

Not applicable.

\section{Funding}

This work was supported by the National Natural Science Foundation of China (No. 31600118). Also, it was supported by Outstanding Youth Plan of Fujian Agriculture and Forestry University (No. xjq201412) and Industry-University Cooperation Project of Universities by Fujian Provincial Department of Science and Technology (CN) (No. 2017Y4002).

\section{Publisher's Note}

Springer Nature remains neutral with regard to jurisdictional claims in published maps and institutional affiliations.

Received: 15 September 2018 Accepted: 31 January 2019

Published online: 11 February 2019

\section{References}

1. Yu J, Cleveland TE, Nierman WC, Bennett JW. Aspergillus flavus genomics: gateway to human and animal health, food safety, and crop resistance to diseases. Rev Iberoam Micol. 2005;22:194-202.

2. Klich MA. Aspergillus flavus: the major producer of aflatoxin. Mol Plant Pathol. 2007:8:713-22.

3. Michailides T, Thomidis T. First report of Aspergillus flavus causing fruit rots of peaches in Greece. Plant Pathol. 2007;56:352.

4. Amaike S, Keller NP. Aspergillus flavus. Annu Rev Phytopathol. 2011;49:107-33.

5. Kensler TW, Roebuck BD, Wogan GN, Groopman JD. Aflatoxin: a 50-year odyssey of mechanistic and translational toxicology. Toxicol Sci. 2011;120:S28-48

6. Gonçalves SS, Cano JF, Stchigel AM, Melo AS, Godoy-Martinez PC, Correa B, Guarro J. Molecular phylogeny and phenotypic variability of clinical and environmental strains of Aspergillus flavus. Fungal Biol. 2012;116:1146-55.

7. Adhikari A, Sen MM, Gupta-Bhattacharya S, Chanda S. Volumetric assessment of airborne fungi in two sections of a rural indoor dairy cattle shed. Environ Int. 2004;29:1071-8.
8. Sepahvand A, Shams-Ghahfarokhi M, Allameh A, Jahanshiri Z, Jamali M, Razzaghi-Abyaneh M. A survey on distribution and toxigenicity of Aspergillus flavus from indoor and outdoor hospital environments. Folia Microbiol. 2011;56:527-34.

9. Zain ME. Impact of mycotoxins on humans and animals. J Saudi Chemi Soc. 2011;15:129-44.

10. Woronin M. Entwicklungsgeschichte des Ascobolus pucherrimus $\mathrm{Cr}$. und einiger Pezizen. Abh Senkenb Naturforsch. 1864;5:344-55 (in German).

11. Buller AHR. The translocation of protoplasm through septate mycelium of certain pyrenomycetes, discomycetes, and hymenomycetes. London: Researches in Fungi, Longmans, Green and Co; 1933. p. 75-167.

12. Reichle RE, Alexander JV. Multiperforate septations, Woronin bodies, and septal plugs in Fusarium. J Cell Biol. 1965;24:489-96.

13. Collinge AJ, Markham P. Woronin bodies rapidly plug septal pores of severed Penicillium chrysogenum hyphae. Exp Mycol. 1985;9:80-5.

14. Jedd $\mathrm{G}, \mathrm{Chua} \mathrm{N}-\mathrm{H}$. A new self-assembled peroxisomal vesicle required for efficient resealing of the plasma membrane. Nat Cell Biol. 2000;2:226-31.

15. Yuan P, Jedd G, Kumaran D, Swaminathan S, Shi H, Hewitt D, et al. A HEX-1 crystal lattice required for woronin body function in Neurospora crassa. Nat Struct Mol Biol. 2003:10:264-70.

16. Dhavale T, Jedd G. The fungal woronin body. In: Howard RJ, Gow NAR, editors. Biology of the fungal cell. 2nd ed. Berlin: Springer; 2007. p. 87-94.

17. Soundararajan S, Jedd G, Li X, Ramos-Pamploña M, Chua NH, Naqvi NI. Woronin body function in Magnaporthe grisea is essential for efficient pathogenesis and for survival during nitrogen starvation stress. Plant Cell. 2004;16:1564-74

18. Maruyama J, Juvvadi PR, Ishi K, Kitamoto K. Three-dimensional image analysis of plugging at the septal pore by Woronin body during hypotonic shock inducing hyphal tip bursting in the filamentous fungus Aspergillus oryzae. Biochem Biophys Res Commun. 2005;331:1081-8.

19. Beck J, Ebel F. Characterization of the major Woronin body protein HexA of the human pathogenic mold Aspergillus fumigatus. Int J Med Microbiol. 2013;303:90-7.

20. Juvvadi P, Maruyama JK, Kitamoto K. Phosphorylation of the Aspergillus oryzae Woronin body protein, AoHex1, by protein kinase C: evidence for its role in the multimerization and proper localization of the Woronin body protein. Biochem J. 2007:405(Pt 3):533.

21. Son M, Lee K-M, Yu J, Kang M, Park JM, Kwon S-J, et al. The HEX1 gene of Fusarium graminearum is required for fungal asexual reproduction and pathogenesis and for efficient viral RNA accumulation of Fusarium graminearum virus 1. J Virol. 2013;87:10356-67.

22. Han P, Jin FJ, Maruyama J, Kitamoto K. A large nonconserved region of the tethering protein leashin is involved in regulating the position, movement, and function of woronin bodies in Aspergillus oryzae. Eukaryot Cell. 2014;13:866-77.

23. Liang LM, Gao H, Li JZ, Liu L, Liu ZH, Zhang KQ. The Woronin body in the nematophagous fungus Arthrobotrys oligosporais essential for trap formation and efficient pathogenesis. Fungal Biol. 2017;121:11-20.

24. Tenney K, Hunt I, Sweigard J, Pounder J, MCClain C, Bowman EJ, et al. Hex-1, a gene unique to filamentous fungi, encodes the major protein of the woronin body and functions as a plug for septal pores. Fungal Genet Biol. 2000;31:205-17.

25. Yu J. Current understanding on aflatoxin biosynthesis and future perspective in reducing aflatoxin contamination. Toxins. 2012;4:1024-57.

26. Yu J, Chang PK, Ehrlich KC, Cary JW, Bhatnagar D, Cleveland TE, et al. Clustered pathway genes in aflatoxin biosynthesis. Appl Environ Microbiol. 2004;70:1253-62.

27. Tiwari S, Shankar J. Integrated proteome and HPLC analysis revealed quercetin-mediated inhibition of aflatoxin B1 biosynthesis in Aspergillus flavus. 3 Biotech. 2018:8:47.

28. Markha P, Collinge AJ. Woronin bodies of filamentous fungi. FEMS Microbiol Rev. 1987:46:1-11.

29. Lim DB, Hains $P$, Walsh B, Bergquist $P$, Nevalainen $H$. Proteins associated with the cell envelope of Trichoderma reesei: a proteomic approach. Proteomics. 2001;1:899-909.

30. Momany M, Richardson EA, Van Sickle C, Jedd G. Mapping Woronin body position in Aspergillus nidulans. Mycologia. 2002;94:260-6.

31. Tang J, Li YY, Fu KH, Yuan X, Gao SG, Wu Q, et al. Disruption of hex1 in Trichoderma atroviride leads to loss of Woronin body and decreased tolerance to dichlorvos. Biotechnol Lett. 2014;36:751-9. 
32. Son $M$, Choi $H$, Kim K-H. Specific binding of Fusarium graminearum Hex1 protein to untranslated regions of the genomic RNA of Fusarium graminearum virus 1 correlates with increased accumulation of both strands of viral RNA. Virology. 2016;489:202-11.

33. Nie XY, Yu S, Qiu MG, Wang XN, Wang Y, Bai YH, et al. Aspergillus flavus SUMO contributes to fungal virulence and toxin attributes. J Agric Food Chem. 2016;64:6772-82.

34. Zhuang Z, Lohmar JM, Satterlee T, Cary JW, Calvo AM. The master transcription factor $m t f A$ governs aflatoxin production, morphological development and pathogenicity in the fungus Aspergillus flavus. Toxins. 2016;8:E29.

35. Szewczyk E, Nayak T, Oakley CE, Edgerton H, Xiong Y, Taheri-Talesh N. Fusion PCR and gene targeting in Aspergillus nidulans. Nat Protoc 2006;1:3111-20
36. Yang KL, Liang LL, Ran FL, Liu YH, Li ZG, Lan HH, et al. The DmtA methyltransferase contributes to Aspergillus flavus conidiation, sclerotial production, aflatoxin biosynthesis and virulence. Sci Rep. 2016;6:23259.

37. Zhang F, Xu GP, Geng LP, Lu XY, Yang KL, Yuan J, et al. The response regulator AflSkn7 contributes to morphological development, stress response, and pathogenicity in the fungus Aspergillus flavus. Toxins. 2016;8:202.

38. Livak KJ, Schmittgen TD. Analysis of relative gene expression data using real-time quantitative PCR and the 2(-Delta Delta $C(T)$ ) method. Methods. 2001;25:402-8
Ready to submit your research? Choose BMC and benefit from:

- fast, convenient online submission

- thorough peer review by experienced researchers in your field

- rapid publication on acceptance

- support for research data, including large and complex data types

- gold Open Access which fosters wider collaboration and increased citations

- maximum visibility for your research: over 100M website views per year

At BMC, research is always in progress.

Learn more biomedcentral.com/submissions 